\title{
BÉLA BALÁZS, BÉLA BARTÓK ET LE THÉÂTRE HONGROIS. LE CAS DU PRINCE DE BOIS
}

\author{
JEAN-FRANÇOIS BOUKOBZA \\ Professeur au Conservatoire national supérieur de danse de Paris \\ jf.boukobza@cnsmdp.fr
}

\begin{abstract}
Le Prince de bois de Bartók n'a pas connu le succès escompté lors de sa création, il correspondait peu aux attentes du public, bien que son intrigue de base fût similaire à celles d'autres ballets du moment, comme Coppelia. L'auteur du livret, Béla Balázs, était proche des milieux d'avant-garde, autour du Cercle du dimanche de Georg Lukács, mais son intention de plonger dans les profondeurs du drame humain en s'intéressant moins aux conditions sociales le tenait éloigné, non seulement de la bourgeoisie, mais aussi des mouvements d'émancipation. L'oeuvre peut être envisagée sous l'angle de la psychanalyse des contes de fée. Une analyse de Ferenczi met en évidence l'idée de la toute-puissance du moi. Sur un fond autobiographique, Bartók a donné une forme musicale à cette pièce illustrant la difficulté des relations entre l'homme et la femme. Il répond au livret de Balázs en combinant notamment plusieurs thématiques musicales (des danses) illustrant l'opposition entre l'authentique et l'artificiel, ainsi que la progression dramatique de l'amour, du désespoir et du rituel initiatique.
\end{abstract}

Mots-clefs : Béla Bartók, Béla Balázs, avant-garde et modernité, psychanalyse du conte

Un prince tombe amoureux d'une princesse retenue au cœur d'une forêt par une fée gardienne de la nature. Il tente de traverser bois et étangs pour la rejoindre, mais ne peut franchir les obstacles naturels qui se dressent contre lui. Pour attirer l'attention de la jeune femme, il construit une poupée de bois à sa propre effigie et l'agite devant lui. Le stratagème échoue : la Princesse s'éprend de la marionnette à qui la fée, entre temps, a insufflé la vie. Le Prince est désespéré. Avec le temps, toutefois, la poupée se disloque, provoquant l'impatience puis l'ire de la jeune femme. Quand celle-ci aperçoit enfin le jeune homme, elle s'éprend de lui. Mais elle connaît l'échec, à son tour, car le Prince est échaudé par ses propres tribulations. Tout de même, elle parvient bientôt à troubler ce dernier de sorte que l'histoire se termine pour le mieux du monde.

Telle est la teneur du texte de Béla Balázs paru dans la revue hongroise Nyugat au mois de décembre 1912. Le thème n'est pas nouveau. Le sujet de la poupée de bois amenée à la vie a déjà constitué le fond de nombreux ballets tels Casse-Noisette de Tchaïkovski, Coppelia de Delibes ou Petrouchka de Stravinsky. Le scé- 
nario du Prince de bois partage en outre quelques ressemblances avec l'Oiseau de feu ou le Rossignol, comme l'union entravée par un esprit surhumain ou l'opposition avec le mécanique et l'artificiel.

Si le ballet s'inscrit ainsi à la suite d'autres oeuvres, il semble s'insérer plus difficilement au sein de la vie théâtrale hongroise du début de siècle, et paraît peu représentatif de ce que l'on peut applaudir sur la scène budapestoise. Malgré son appartenance à différents cercles - celui des poètes liés à la revue Nyugat et celui du groupe Thalia de György Lukács - Balázs demeure un artiste relativement isolé dans le monde littéraire hongrois du début du $\mathrm{XX}^{\mathrm{e}}$ siècle. Son art est jugé abstrait, spéculatif, trop " inféodé » à la culture allemande - attaques qui masquent souvent des critiques dont l'antisémitisme constitue le fond réel.

Le cas du Prince de Bois est révélateur. La pièce est peu représentative du répertoire donné au sein des principaux théâtres de la capitale. Elle ne reflète pas non plus les courants dominants de la littérature hongroise même si les thèmes qui y sont développés peuvent en paraître issus.

Traitant de la difficulté des rapports humains et du conflit entre l'artiste et sa création, le livret ne pouvait toutefois que séduire Bartók, dont les ouvrages attestent du même intérêt pour ces questionnements et dont l'œuvre se situe au cœur d'une modernité hongroise revendiquée.

\section{L'état des scènes hongroises}

Le théâtre hongrois semble régi par trois veines dominantes au début du siècle : la comédie, à laquelle on peut adjoindre les pièces de cabaret et les opérettes ; le théâtre littéraire et celui d'avant-garde. La notion d'un " théâtre hongrois » est liée à différentes conceptions : l'engagement pour la magyarisation, qui bénéficie du soutien des milieux officiels; la critique de l'ancien système et la promotion de nouveaux modes de vie; le néo-conservatisme, qui révèle une scission à l'intérieur même de la bourgeoisie entre les propriétaires terriens, conservateurs et défenseurs d'une culture exclusivement nationale (cf. les pièces de Jenő Huszka : A bob Herceg - le prince Bob), et la moyenne bourgeoisie, juive, cosmopolite, de langue hongroise ou allemande, appréciant autant les comédies pleines d'esprit de Ferenc Molnár que les opérettes d'Imre Kálmán'.

La vie théâtrale est liée essentiellement à trois lieux, eux-mêmes associés à des répertoires spécifiques : le Théâtre de la Gaieté (Vígszinház), dédié depuis 1896 aux vaudevilles et aux comédies ; le Théâtre du Roi (Király Színház), fondé en 1903 et où domine l'opérette ; le Théâtre Hongrois (Magyar színház) où sont donnés des pièces de salons, des vaudevilles et des comédies françaises. Le genre le plus prisé demeure toutefois l'opérette, où brillent les compositeurs hongrois tels Jenő Huszka (Le Prince Bob, 1902 : La baronne Lili 1919), Ferenc Lehár 
(Le Comte de Luxembourg, 1910 ; Eva, 1912), Albert Szimrai (Noble Miszka, 1913) ou Imre Kálmán (L’invasion tatare, 1908). La Veuve joyeuse de Lehár a été jouée 150 fois à Budapest puis 400 fois à Vienne. La Princesse Csardas de Kálmán a été représentée à Budapest en 1916, au plus fort de la guerre, obtenant un succès sans précédent et presque indécent au vu des circonstances. " Le monde entier retentit de deux choses : du grondement des canons et du succès de la Princesse Csardas » écrit alors un critique viennois ${ }^{2}$. Genre populaire, l'opérette permet de réunifier un public citadin fortement cloisonné. L'action, où il n'est question que d'ascension sociale et de bonheur à prendre au premier degré, la musique pleine de vie, les décors somptueux, les costumes scintillants, les danses animées et les emprunts aux musiques populaires (chansons de cabaret, valse, ragtime, chansons) gagnent aisément les faveurs du public.

Une comédie renouvelée - «moderne »- commence toutefois à apparaître, illustrée par Dezső Szomory, Ernő Szép ou Milan Füst. Ses sujets traitent de la condition de l'artiste (Hermelin, 1916 ; Chère petite, 1912 de Szomory), des angoisses comiques de la bourgeoisie de Budapest (Füst : Le Pianon, La Tante Mali), du milieu social et ouvrier, ou de la question de la communication entre les femmes et les hommes (Füst : Les Malheureux, 1915). Les thèmes d'actualité, les pièces à thèse, les drames politiques et les sujets sociaux liés au théâtre « littéraire » se développent également dans les pièces de Viktor Rákosi ( $\mathrm{LaClo}$ che muette, 1905), Hugo Csergő (Le Noble chevalier), Imre Földes (Les Soldats de l'Empereur) ou Melchior Lengyel, futur librettiste du Mandarin merveilleux de Bartók (Typhon, 1907). Ferenc Herczeg (Les Brigadiers d'Ocskay, 1901) ou Dezső Szomory (L'Empereur Joseph II) promeuvent les pièces à caractère historique, tandis que Lajos Kassák, Tibor Déry ou Ödön Palasovszky s'apprêtent à développer un théâtre avant-gardiste dont les réalisations importantes verront le jour après la guerre (Zsigmond Remenyik : Sang, 1921 ; Sandor Barta : Igen ; János Lékai : Jean, l’homme, 1921 ; Tibor Déry : le Bébé géant 1926, Janos Macza : Le Matou noir, 1921).

Le Prince de bois ne s'inscrit dans aucune de ces veines. Par son sujet comme par son traitement, la pièce se situe en marge de la vie théâtrale hongroise dont elle se montre peu représentative, à l'image de son auteur, Béla Balázs.

\section{Créer le drame hongrois moderne}

Reconnu aujourd'hui comme un théoricien important du cinéma, Béla Balázs (1884-1948) entre dans la vie littéraire hongroise à la fin de la première décennie du XIX ${ }^{\mathrm{e}}$ siècle, publiant des poèmes, des critiques et de petits travaux pour des journaux de Budapest. En 1908, il collabore à la revue Nyugat, pour laquelle il écrit un nombre important d'articles. Il rédige des poèmes lyriques, des nouvelles 
ou des contes dont l'intrigue se déroule souvent dans un milieu oriental ou un cadre exotique.

S'il participe à différents cercles, il reste toutefois isolé dans le panorama littéraire hongrois du début de siècle. Dès le commencement de sa carrière, il est considéré comme un « marginal » auquel on adresse trois reproches principaux : celui d'être Juif, d'écrire de manière trop abstraite, puis de demeurer attaché à une culture allemande alors fortement combattue par les nationalistes magyars. Né de parents allemands immigrés (Balázs est un pseudonyme ; son nom réel est Herbert Bauer), il incarne l'érudit germanique de fin de siècle intéressé par les ouvrages d'Ibsen, Hofmannsthal, d'Annunzio, Strindberg ou Maeterlinck. À l'université de Budapest, dont il suivait les cours en même temps que ceux du prestigieux collège Eötvös, il obtient un doctorat en philologie allemande pour une thèse intitulée « Le pan tragique chez Friedrich Hebbel, comme résultat de la vision du monde romantique ». Ses amis et les personnalités qu'il admire - György Lukács, Max Weber et George Simmel - sont eux-mêmes des intellectuels de culture allemande. Sa fréquentation du Cercle Thalia fondé par Lukács, enfin, est décisive pour l'évolution de ses idées esthétiques et philosophiques ainsi que sa connaissance des œuvres de Hauptmann, Gorki ou Tchékhov.

La critique devient si virulente à son endroit qu'elle contraint Lukács à prendre la plume pour le défendre dans une série d'essais assemblés en 1918 sous le titre de Béla Balázs et ceux qui ne veulent pas de lui. Le volume paraît au moment où est donnée la première représentation à Budapest du Château de Barbe-Bleue de Bartók (dont Balázs a signé le livret). Le poète et le musicien se sont rencontrés pour la première fois en 1906, à l'occasion d'un voyage dans la Hongrie rurale. Une collaboration a commencé malgré la froideur ou la distance relative des premières relations. Le Château de Barbe-Bleue est porté à la connaissance du Comité des Beaux-arts, qui rejette la partition, suivi en cela par l'Opéra de Budapest. Le public budapestois n'est en effet pas encore prêt pour le théâtre proposé par les deux hommes - une scène influencée par le drame romantique allemand, le théâtre symboliste franco-belge (notamment Maeterlinck), les pièces de Strindberg et de Ibsen.

L'ambition de Balázs, qui estime avec Lukács que le théâtre budapestois est passéiste, est de créer un drame hongrois nouveau, «moderne », répondant à un idéal différent de celui des pièces de son temps : ni opérette, ni farce visant l'effet scénique, ni comédie sociale, ni drame politique, mais des œuvres se voulant tout à la fois originales, mystérieuses et poétiques. 


\section{Les orientations esthétiques : la revendication d'un théâtre de l'intériorité}

Plutôt que des rebondissements, des coups de théâtre et des conflits extérieurs, la volonté de Balázs est de présenter « les processus inexplicables de l'âme » et d'aspirer à une intériorisation du drame ${ }^{4}$. En 1912, il fait publier sous le titre de Mystères trois pièces achevées au cours des mois précédents : Le Château de BarbeBleue, Le Sang de la Vierge et La Fée. Ecrites en vers, ces dernières «n'ont rien en commun avec les mystères du Moyen Age $^{5} »$ : le terme signifie ici les aspects énigmatiques de l'âme et des rapports humains, dont les plus importants sont l'amitié et l'amour. L'action est purement abstraite : c'est le rapport entre les personnes, leurs caractères, leur psychologie ou leur dualité qui forment le mouvement dramatique.

Balázs cherche à concilier le monde des passions définissant l'univers de la ballade (l'amour, la haine, la jalousie qui poussent irrésistiblement les personnages vers leur destin) et les clés symboliques du conte, où le drame cèle un message à interpréter. Dans le Château de Barbe-Bleue comme dans le Prince de bois, le cadre est atemporel. Les personnages sont conçus comme des entités symboliques : Judit et Barbe-Bleue incarnent les principes féminins et masculins ; le Prince et la Princesse représentent deux adolescents découvrant l'amour. Les éléments du décor sont chargés de significations : le chemin symbolise la distance qui sépare les êtres ou les sexes et il peut également dessiner le destin de l'homme ou ses objectifs; le château est tour à tour un lieu clos, un espace spirituel et intime ou l'âme elle-même ; la forêt figure le monde extérieur et les obstacles à traverser. La lecture est orientée vers la tragédie des rapports masculins et féminins. Amour et connaissance sont des qualités qui s'excluent lorsque l'une domine et prend le pas sur l'autre. L'unité du couple réside dans la possibilité de respecter un équilibre entre les deux, mais elle est anéantie lorsque la quête de l'identité prime. La thématique évoque bien sûr les opéras de Wagner (Le Vaisseau fantôme, Lohengrin, Parsifal) ; c'est leur traitement qui est innovant.

\section{Entre Maeterlinck et Ady}

Dès le début de ses activités littéraires, Balázs s'est senti attiré à la fois par le romantisme allemand et par le symbolisme français. Il connaît parfaitement les pièces de Maeterlinck, qu'il mentionne plusieurs fois dans ses notes comme étant " un auteur dramatique de première importance ${ }^{6}$ ». En 1908, il écrit dans la revue Nyugat un essai sur Maeterlinck, notant son aptitude à sonder les secrets de l'âme humaine. Le Château de Barbe-Bleue et le Prince de bois racontent elles aussi des histoires dont les héros sont silencieux et immobiles, retenus dans des châteaux qui sont des prisons mentales. 
Du dramaturge belge, Balázs apprécie spécifiquement les pièces courtes en un acte, tels L'intruse (1890), Les Aveugles (1890) ou Intérieur (1894). Il en apprécie le refus du naturalisme, le rythme statique et le ton intériorisé : des personnages en attente d'un événement funèbre ou tragique ; un drame silencieux ; un intérêt pour le psychologique ; une atmosphère onirique où les déplacements sont infimes et l'action raréfiée ; une importance nouvelle accordée aux jeux de regards ; un contrepoint du son et du silence ; des phrases brèves, parfois inachevées; des dialogues troués de vides ${ }^{7}$. Le langage se montre inopérant à exprimer ce qui relève de l'intuitif, de l'inné, du naturel. L'opposition de l'obscur et du lumineux fait ressortir le destin tragique des protagonistes, enfermés dans une destinée sans perspective. Les personnages, peu individualisés, sont des figures ordinaires reliées à de simples fonctions ${ }^{8}$ : le Père, le Vieillard, l'Etranger, la Femme, la Fiancée. Les lieux sont peu définis, le temps rarement indiqué. La mort est le personnage principal. Elle n'est ni brutale ni effrayante mais perçue comme celle qui détient le secret dernier, qui possède la clé du mystère commandant toute vie.

Proche de Maeterlinck dans son goût du mysticisme, Balázs se plonge dans un univers dramatique encore plus abstrait, plus poétique et stylisé. Si son langage scénique reste ancré dans le quotidien, les symboles sont moins aisés à décrypter, la lecture plus ambivalente ou polysémique. Les thématiques portent essentiellement sur les rapports homme/femme, l'impossibilité du couple ou la nature de l'amour - et pas seulement sur la mort, comme chez l'écrivain francophone.

À l'influence de Maeterlinck se combine celle d'Endre Ady, dont le recueil intitulé Poèmes nouveaux, paru en 1906, a fait l'effet d'une bombe. Considéré comme un "phénomène " historique et politique aussi bien que littéraire, Ady ne poursuit qu'un seul dessein : faire entrer la Hongrie archaïque dans l'occident moderne. Tout ce qui est arriéré, « oriental », bureaucratique ou conservateur le révulse. Ses écrits en prose, publiés dans les colonnes de Nyugat, inaugurent un nouveau style, où l'érotisme et l'amour charnel sont définis comme étant les plus hautes expériences. Ses nouvelles, comme ses récits, parlent de l'amour sous la forme de souvenirs d'adolescence ou d'expériences de l'homme mûr. La condition féminine est observée d'une manière à la fois poétique et clinique. La sensualité, la liberté sexuelle, l'idée de l'égalité entre l'homme et la femme ou la détermination sociale jouent un rôle de premier plan.

Marquées par cette influence, les premières pièces de Balázs (Dr. Szélpál Margit, Jeunesse mortelle) évoquent à leur tour l'antagonisme masculin/féminin, ou ce qui est vécu comme tel. Ses héroïnes sont « des femmes modernes qui se voient contraintes de choisir entre la profession et la famille ${ }^{9}$, l'acceptation de la domination masculine ou l'isolement, l'abnégation ou le sacrifice, et ne trouvent souvent une résolution que dans la mort ${ }^{10}$. Ses premières pièces ne rencontrent pas le succès. Le croisement d'influences y est sans doute trop dense pour le public de 1910, comme est également trop neuve l'idée d'un théâtre totalement statique et intériorisé. 


\section{La version littéraire du Prince de bois : le conte et son interprétation}

La première version du Prince de bois emprunte son format général au conte, dont elle conserve les attributs et la structuration : un récit court relevant de la littérature narrative ; une histoire inventée ayant le statut d'imagination et de fantaisie ; des personnages sans passé et sans identité ; une histoire reposant sur des anomalies et des invraisemblances admises. Pourquoi la Princesse, par exemple, qui a vu le Prince en chair et en os, n'est-elle pas immédiatement séduite par lui, alors qu'elle l'est par le bâton portant sa couronne, ses vêtements et ses cheveux ? Pourquoi la Fée, pourtant si active dès le début, ne cherche-t-elle pas à séduire définitivement le Prince dont elle s'est également amourachée ? Pourquoi renonce-t-elle si facilement?

$\mathrm{Au}$ conte, le récit emprunte également la structure par trois liée au genre et révélée notamment par Bruno Bettelheim dans Psychanalyse des contes de fées : trois personnages principaux, trois collines correspondant aux personnages, par trois fois, injonctions de la Fée à la Princesse de se retirer ${ }^{11}$, trois lignes de forêt à traverser, trois tentatives pour séduire la Princesse ${ }^{12}$. Balázs joue également avec les conventions, en réunissant tous les éléments traditionnels des contes - des châteaux pourvus de tourelles, une colline, une forêt "sauvage et immense », un chemin menant vers un ruisseau, une pierre symbolisant le destin - mais en les érotisant passablement. Les trois collines sont décrites comme ayant des « formes arrondies », le ruisseau est " modelé comme les seins de cent femmes couchées », les vagues possèdent des formes « amples, rondes, généreuses », les arbres de la forêt « se sont changés en femme », les vagues forment un « corps palpitant».

Les personnages incarnent différents stades de l'amour mais également différentes étapes de la maturité sexuelle. La Fée joue le rôle d'une mère, tour à tour tyrannique et séduisante, castratrice et attirante. La Princesse est une jeune fille nubile qui commence à ressentir le désir. Le Prince en constitue le double masculin (mêmes attributs, même rang social) mais sa maturité plus prononcée lui fait déjà quitter l'état de l'enfance. Le Bâton symbolise la virilité, la pulsion sexuelle (cf. l'agitation du Prince) et sans doute le désir de perdre le pucelage, d'abandonner ce qui ligote le Prince à l'enfance. Si la maturité sexuelle paraît plus forte chez l'homme que chez la femme, l'image de la Princesse commençant à filer (« travailler dans un moment pareil !») laisse toutefois deviner un mélange d'innocence, d'immaturité et de désir lié à l'image sexuelle du rouet... Le Prince devant courir les grands chemins, enfin, est la représentation même de l'adolescent quittant l'enfance.

Pour l'esprit spéculatif de Balázs, la signification du conte est plurielle et la surdétermination de règle. Les niveaux de lecture s'entrecroisent sans que l'un domine, mais sans que l'autre soit non plus longtemps absent. La rivalité mère/ 
fille/Fée fait fuir la princesse, quitte à manier la menace («Attends un peu !»). Les mondes masculins et féminins semblent définitivement séparés, à travers l'image des deux châteaux isolés (celui du Prince, celui de la Princesse). La série d'épreuves à traverser ne sert pas seulement à tester l'authenticité de l'amour, la force du désir, le passage au stade adulte ou l'assomption des choix, mais évoque aussi la rupture de l'éden prénatal et l'intégration, par les deux adultes en devenir, du principe de réalité.

La satisfaction immédiate des désirs, liée à la libido masculine et à l'omnipotence de l'enfant-roi, passe par une initiation : la forêt qui mène vers la Princesse, et, symboliquement, vers la réalisation de l'union. La quête initiatique exige de savoir résister aux tentations - les arbres déguisés en femmes - de même qu'à l'aspiration à une vie de plaisir et de débauche que repousse alors le Prince. Son parcours lui enseigne l'échec et lui en fait prendre conscience. Sa réflexion est alors insuffisante. Lorsqu'il sera « mûr », c'est la Princesse qui devra, à son tour, subir des épreuves - considérer les vraies valeurs, rejeter le superficiel, mesurer l'être et le paraître.

Ce dernier aspect est le point nodal du conte. Si le Prince est prêt à renoncer à ses attributs pour séduire, La Princesse, encore infantile, se montre, elle, horrifiée par la vision du Prince chauve, laid et sans parure. Elle découvre progressivement les illusions et les désillusions de l'amour, et fait l'expérience d'une nouvelle forme d'apprentissage sur la vérité du monde. La séduction du pouvoir, à travers l'image du Prince enivré de gloire, constitue la dernière épreuve : la possibilité de se perdre dans un monde de débauche et de fausses valeurs. En faisant du Prince un roi, la Fée laisse deviner la perte fictive du pucelage et le passage symbolique vers le stade adulte.

Un autre thème domine, celui de l'artiste et sa création. Balázs pose à la fois la question de l'œuvre se dérobant au créateur et celle du du « Beau artificiel » comme potentiellement supérieur au Beau réel. En ce sens, il rejoint les questionnements de l'avant-garde hongroise concernant le statut et le rôle de l'artiste. Un grand nombre de nouvelles sont notamment consacrées à ce sujet, chez Csáth (Suicides d'artistes), Kosztolányi (La Disparition), Dezső Szomory (Bella), Milan Füst (Le Révolté) ou Karinthy (Voyage autour de mon crâne). Le concept de sublimation, dont Freud vient de révéler le mécanisme, et le problème de l'artiste confronté à sa propre création sont au cœur du Prince de bois : «Le Prince de bois symbolise l'acte créateur de l'artiste, qui s'investit totalement dans son œuvre jusqu'à ce qu'il ait achevé quelque chose de complet, d'étincelant et de parfait. L'artiste lui-même, cependant, en ressort volé et appauvri. Je pensais à cette tragédie profonde (quoique commune), lorsque la création devient la rivale de son créateur et génère une situation à la fois douloureuse et héroïque, où la femme préfère le poème au poète, la peinture au peintre » écrit Balázs lui-même ${ }^{13}$. 
Dans un numéro de Nyugat, Anna Lesznai, belle-sœur de Balázs, publie quelques réflexions stimulantes sur la psychologie du conte. Elle critique notamment fortement le fait que les disciples de Freud voient uniquement dans le conte l'accomplissement d'un désir sexuel, ce qui lui vaut une réponse de Ferenczi comme quoi les Freudiens n'ont jamais simplifié la vie psychique jusqu'à l'assimiler entièrement à la sexualité ${ }^{14}$. Dans l'article " Le Développement du sens de réalité et ses stades », le grand psychanalyste traite du remplacement du principe de plaisir par l'adaptation à la réalité - autrement dit, par la désillusion de l'enfant qui doit intégrer le principe de réalité ${ }^{15}$. Ferenczi signale à cette occasion que le genre du conte représente un retour à la toute-puissance du moi - sans limitation. Le sujet touche de près Le Prince de bois.

Dans les contes, explique-t-il, les fantasmes de toute-puissance continuent de régner sans partage et peuvent apporter un soulagement au lecteur en devenir. « Dans la réalité, nous sommes faibles, les héros du conte seront donc forts et invincibles ; nous sommes limités par le temps et l'espace dans notre activité et notre savoir : dans les contes, on vit éternellement, on est dans mille endroits à la fois, on prévoit l'avenir et on connaît le passé. La pesanteur, la dureté et l'impénétrabilité de la matière constituent à tout instant des obstacles sur notre chemin, mais l'homme, dans les contes, a des ailes, son regard perce les murs, sa baguette magique lui ouvre toutes les portes. La réalité est un dur combat pour l'existence : il suffit dans le conte de prononcer quelque parole magique : "Petite table, couvre-toi !" Nous vivons dans la crainte perpétuelle d'être attaqués par des bêtes dangereuses ou des ennemis féroces : le manteau magique du conte permet toutes les transformations et nous met rapidement hors d'atteinte. Combien il est difficile dans la réalité d'atteindre à un amour qui comble tous nos désirs : le héros de conte est irrésistible, ou bien il séduit d'un geste magique.

Ainsi le conte, dans lequel les adultes racontent si volontiers à leurs enfants leurs propres désirs insatisfaits et refoulés, donne en vérité une représentation artistique extrême de la situation perdue de toute-puissance ${ }^{16}$. »

L'article de Ferenczi permet de jeter une lumière nouvelle sur Le Prince de bois. Le Prince ne peut satisfaire immédiatement ses désirs sexuels et doit intégrer le principe de réalité : attirer l'attention de l'autre, le séduire, connaître l'échec, débuter un itinéraire et un apprentissage amoureux. Il s'agit bien d'un conte, mais nullement d'un conte de fée, car il ne préserve pas la toute-puissance du héros. Pire, les deux personnages principaux perdent leurs illusions de toute-puissance. Le Happy end referme l'intrigue, certes, mais après une longue série d'épreuves, de désespoirs, de désillusions et de brûlures.

C'est précisément l'aspect favorisé par Bartók dans sa mise en musique. Le compositeur semble avoir accentué le désespoir du Prince et favorisé, dans son oeuvre, le moment du conte où le protagoniste est le plus durement confronté au principe de réalité. 


\section{Le ballet}

L'orchestration est achevée en 1917, mais Bartók en a commencé l'esquisse au mois d'avril 1914. Son origine remonte même au mois de décembre 1912. Le 30 de ce mois, les Ballets Russes de Serge Diaghilev donnent en création hongroise à l'Opéra de Budapest L'Oiseau de feu d'Igor Stravinsky. Le succès obtenu incite l'Opéra à commander à Bartók un ballet. Le premier réflexe du musicien est le refus, car le rejet de la partition du Château de Barbe-Bleue l'a profondément blessé. Pourtant, c'est en raison même de ce refus que l'idée fait finalement son chemin :

« C'est peut-être étrange, mais j' avoue que l'impulsion pour l'écriture de ce ballet m'est venue du refus de mon opéra en un acte Le Château de Barbe-Bleue. On le sait, cet ouvrage a échoué lorsqu'il fut présenté à un concours d'opéra. Le plus grand obstacle à sa mise en scène, c'était que l'intrigue se limite au pur conflit psychique entre deux personnes, la musique se bornant elle aussi à le représenter de façon abstraitement simple. Il ne se passe rien d'autre sur la scène. J'aime tellement mon premier opéra que, lorsque j'ai reçu de Béla Balázs l'argument du ballet, j'ai tout de suite pensé : par son aspect spectaculaire, par ses successions d'événements hauts en couleur, riches et variés, le ballet ouvrira la possibilité que mes deux œuvres soient portées à la scène lors d'une même soirée. Il est inutile de souligner, je crois, que je me sens désormais tout aussi proche du ballet que de mon opéra.

J'ai entamé la composition du ballet avant la guerre, puis je l'ai interrompue pendant longtemps. J'ai traversé beaucoup d'inquiétudes. Lors de la saison dernière István Strasser a présenté mon œuvre symphonique intitulée Deux Portraits, dont j'entendais le second (Difforme) pour la première fois à l'orchestre. C'est alors que j'ai eu l'inspiration pour continuer la partition du Prince de bois et, de fait, je l'ai achevée en peu de temps.

La musique du ballet est élaborée dans le genre symphonique : un poème symphonique sur lequel on danse. On peut y distinguer clairement trois parties, dans lesquelles il y a aussi, toutefois, des articulations à plus petite échelle. La première partie va jusqu'au pas de deux du pantin de bois et de la princesse. La deuxième, bien plus calme que la première, a les caractéristiques d'un mouvement central et dure jusqu'au retour du pantin de bois. La troisième partie est en fait la reprise de la première, mais en renversant l'ordre des composantes, ce que l'argument, du reste, requiert par sa nature ${ }^{17}$. »

Bien que concis, le texte de Bartók offre beaucoup de renseignements. En premier, lieu, ce qui pourrait apparaitre comme une divergence avec la conception intériorisée de Balázs : l'idée que le ballet pourrait avoir un « aspect spectaculaire » en offrant des événements « hauts en couleurs, riches et variés ». En second lieu, l'idée de lier le Prince de bois et Le Château de Barbe-Bleue et les 
réunir dans la même soirée. Zoltán Kodály a révélé ce qui peut constituer le fonds de la pensée de Bartók : assimiler les œuvres à un vaste Verbunkos (une forme typique de la musique hongroise où se succèdent une partie lente, de nature improvisée et intériorisée, et une partie rapide, de plus en plus exaltée ; ici, l'opéra et le ballet, successivement) ${ }^{18}$.

La présentation de Bartók apporte d'autres précisions : l'attache avec les Portraits pour orchestre, et notamment le second, généralement intitulé Grotesque. Ce lien révèle les conséquences encore perceptibles de la rupture avec Stefi Geyer, une violoniste avec laquelle Bartók avait eu une liaison et dont la séparation avait été particulièrement douloureuse. La conception amère de l'amour exposée tout au long du Prince de bois prend alors des résonances clairement autobiographiques.

Sur un dernier plan, enfin, Bartók dévoile la construction ternaire du Prince de bois, ainsi que l'idée d'une forme en arche. Le palindrome est très dissymétrique toutefois. La première partie, la plus longue, atteint la demi-heure environ ; les deux autres sont nettement plus courtes - une douzaine de minutes pour la deuxième, dix minutes pour la dernière. La première partie (chiffres $0 / 120$ ) expose les fondements de l'action, caractérise les protagonistes et mène vers une première crise. La deuxième (chiffres 120/142) montre le désespoir existentiel du Prince et se referme sur un nouveau sommet émotionnel. La troisième (chiffre $142 /$ fin) résout les conflits et propose un retour en ordre inversé (et passablement varié...) des éléments de la première. L'ensemble est structuré en sept danses entrecoupées de "scènes 》- de passages dévolus à l'avancée de l'action. Le fondement de l'intrigue est mis en musique dans un épisode de forme ternaire qui place en son centre - au cœur même du ballet donc - le désespoir du Prince :

Prélude : Do M acoustique

Postlude : Do M

Danse 1 : chiffre 11 : Si b M

(Danse de la princesse dans la forêt)

Danse 2 : 23 : do M (locrien, octotonique) (Danse des arbres)

Danse 7 : 167 : V de Do M (Danse de la Princesse effrayée)

Danse $3: 39^{+8}:$ Mi m (Danse des vagues)

Danse 6 : 156 : Do M (La Princesse et le Prince)

Danse $4: 88^{+4}:$ La m (La Princesse et la Poupée)

Danse 5 : 147 : cycle de quintes (Danse de la Poupée)

\section{Désespoir du prince}

Forme aba'

Do m/Do\# m 
La partition est unifiée par la tonalité d'Ut (majeur et mineur) dont la récurrence sert à articuler la grande forme. Le point culminant est situé au ton napolitain (Ut dièse mineur, triomphe du Prince, chiffre 139) - une tonalité liée généralement à un assombrissement du tissu et qui correspond bien, ici, à l'idée d'une dramatisation du discours.

Chaque personnage est défini par un leitmotiv (y compris la Poupée de bois) auquel s'ajoute celui de la "découverte de l'amour »-la réaction du Prince lorsqu'il aperçoit pour la première fois la Princesse. Le motif va jouer un rôle essentiel tout au long de la deuxième partie. Si le motif du Prince (chiffre 19, juste après la fin de la première danse) peut évoquer une chanson hongroise traditionnelle par son ossature pentatonique, son diatonisme, sa tonalité mixolydienne et son architecture interne quaternaire, Bartók n'a intégré dans son ballet aucun thème authentique. Le motif de la Princesse (présenté au chiffre 11) évoque au contraire le thème associé à Judith dans Le Château de Barbe-Bleue par sa mélodie ondulante, son chromatisme sensuel, et son habillage délicat réalisé grâce au timbre doux de la clarinette.

Quelques danses retiennent particulièrement l'attention. Celle de la Princesse joint des figures de triolets issues du Verbunkos et associant la jeune fille à une musique monodique et modale, liée au répertoire hongrois. La Danse des arbres est fondée sur une animation permanente du tissu : des changements multiples de tempo, une tonalité fortement chromatisée, une figure des timbales divisant la mesure à $3 / 4$ de manière irrégulière, un fugato terminal.

La Danse des vagues évoque à son tour la musique traditionnelle par la succession de trois mélodies liées au fond magyar. Le pentatonisme latent de la première (chiffre 42) renvoie au fond traditionnel. La deuxième (chiffre 45, Piu mosso) intègre une structure quaternaire type. La troisième (chiffre 47) est une mélodie sans reprise, en mode de Ré sur Do. La danse est également définie par des teintes modales (Mi mineur dorien), des couleurs argentines (célesta, harpe, clarinette en La) et l'intégration de deux parties saxophones (alto et ténor). La Danse de la Poupée, enfin, est un scherzo animé qui n'est pas sans rappeler les épisodes «mécaniques » omniprésents dans la musique de Stravinski.

\section{La lecture bartokienne : convergence ou divergence avec Balázs?}

Tout au long de la partition, Bartók s'efforce de répondre avec finesse aux problématiques du livret : différencier l'authentique (le Prince), le superficiel (la Princesse) et l'artificiel (la Poupée) ; préserver l'univers du conte (et du mystère) en combinant un langage accessible et un esprit de distanciation; travailler sur une expressivité directe et poétique... tout en maintenant un style et une esthétique personnels. 
Par rapport à la nouvelle éponyme, le livret, revu par Balázs, est moins développé quant à ses implications. La Fée est présentée comme une sorte de déesse de la nature et son désir pour le Prince est effacé, tandis qu'il constituait un élément important de la version originale dans laquelle il symbolisait la rivalité mère/fille.

Au sein de cette symphonie continue, trois moments sont particulièrement dramatisés par Bartók. Ils permettent de rythmer l'ensemble de l'intrigue tout en faisant ressortir et comprendre la lecture bartokienne du livret, qui confère une emphase claire à la découverte de l'amour et de ses douleurs. L'élément humain prend désormais le pas sur l'aspect symbolique. Du moins, les deux aspects se contrepointent au sein d'une dialectique assez raffinée.

Le premier passage correspond à la vision de la Princesse par le Prince, épisode qui déclenche une brûlure d'amour et un véritable " cri » (chiffre 21). Le tutti fortissimo, la suspension piano molto espressivo et le recours aux timbres expressifs et graves (cor anglais, clarinette, basson, cor) témoignent de la rudesse du choc. Les appels de quartes se répercutent sur les basses et génèrent l'harmonie (chiffre $21^{+2}$ ). Les indications agogiques et métronomiques déclinantes ${ }^{19}$ et les accords gauchis par chromatisme accroissent l'intensité émotionnelle. L'épisode « sonne » comme une lamentation hongroise... dans le style ancien : courbe mélodique descendante, rythme libre, caractère déclamatoire, absence de mètre fixe, cellules pointées, allongement rythmique en fin de phrase, degrés altérés (la dominante, notamment).

Le deuxième moment mis en valeur est la scène correspondant à la mise en mouvement de la Poupée de bois (chiffre 63). Le Prince essaie d'attirer sur lui l'attention de la Princesse en construisant la fameuse poupée, mais il se heurte bientôt à une série d'échecs, la Princesse préférant le pantin (l'artefact) à l'original. Le jeu de séduction donne lieu à une dramaturgie complexe où s'opposent le désespoir croissant du Prince, le charme fortement opérant de la capricieuse et coquette jeune femme, et l'importance grandissante de la Poupée.

Trois thèmes liés à trois types de texture alternent :

- Le thème de l'Amour, entendu précédemment, qui incarne ici le désespoir du Prince par la texture fragmentée et morcelée, les oppositions dynamiques brutales, le chromatisme dense et l'altération progressive du motif fondateur ;

- Le thème de la Princesse, qui engendre des lignes souples, gracieuses, ondulantes ;

- La musique associée à la Poupée (dont le thème dérive de celui du Prince), qui donne naissance à une musique répétitive, fonctionnelle et bientôt mécanique (chiffre 52 sq.) : 
La première section expose les tentatives du Prince pour attirer la Princesse : l'agitation du Prince monté sur une pierre avec un bâton recouvert d'un manteau $\left(62^{+4}\right)$, tandis que la Princesse, indifférente, continue à travailler sans prêter attention aux efforts déployés. Le Prince revêt alors la poupée d'un manteau et d'une couronne (chiffre 67) : la Princesse interrompt son activité pour observer la Poupée, sans dévoiler encore beaucoup d'intérêt. Le troisième essai $\left(72^{+6}\right)$ est le bon : la Princesse, émue, sort de son château et s'approche de la Poupée, avant d'être effrayée par l'apparence misérable du Prince $\left(78^{+3}\right)$.

Le troisième moment est le point culminant du ballet. Bartók y consacre toute la deuxième partie de son ballet (chiffre 120) en montrant le passage du désespoir extrême à la renaissance. L'épisode est conçu selon une petite forme ternaire où le chromatisme dense cède progressivement la place à un tissu diatonique, plus ouvertement tonal. La première section traduit le désespoir du Prince par une musique encore influencée par Wagner et par Strauss : appoggiatures résolues sur d'autres appoggiatures, cordes finement divisées, participation importante des cuivres (tuba, trompette, cor, trombone), nature éruptive du tissu, forme ponctuée d'une série de soubresauts fortissimo (chiffres 124, 124+4, 125). La tonalité mineure, colorée par les teintes modales (locriennes) et les harmonies de neuvième naturelle, renforce la couleur pathétique.

La deuxième section commence à l'entrée de la Fée (chiffre 128). Les sonorités « blanches » (célesta, flûtes sur battements d'octaves à l'aigu, doublures de harpe, cor avec sourdine, trilles cordes, glissandos des harpes) et les éléments de gamme par tons appogiaturée (chiffre 131) traduisent le merveilleux. La Fée anime les objets et les choses, qui se mettent à danser autour du Prince, au moyen de lignes expressives entrelacées en canon. Dans la dernière section (chiffre 132), la Fée couronne le Prince puis le pare d'un manteau de fleurs. Le passage mène vers une apothéose (chiffre 137) où le thème de l'Amour domine. Les colorations de gamme par tons et le mode octotonique (un mode faisant alterner ton et demi-tons) disparaissent pour laisser place à un tissu tonal désormais clair (chiffres 136 et 137). Les progressions chromatiques des cors préparent le point culminant (chiffre 139) sur le thème de l'Amour, en majeur (Do\# majeur). À partir de cet instant, le côté sombre et négatif du ballet se tourne au profit d'un versant positif: «À présent tu seras un Roi régnant sur chaque chose » déclare la Fée (didascalie) : le Prince a surmonté les épreuves et pourra mener sa vie d'adulte.

En intensifiant ces trois moments, Bartók a accentué l'importance du sentiment et montré le point de rupture : lorsque l'on découvre l'émotion de l'amour, mais lorsque l'on en subit aussi (en corollaire ?) les brûlures, la cruauté et la violence. Il a illustré « l'instant fragile » où l'artificiel risque de l'emporter sur l'authentique et détruire le créateur véritable. Il a rendu sensible le moment délicat où le doute, l'instabilité, les vicissitudes trop fortes de la vie et le risque de dérive se manifestent et submergent l'individu envahi par le chagrin au point 
de pouvoir le détruire. Peu d'auteurs ont su traduire avec autant de délicatesse le désarroi éprouvé devant la découverte de la vérité du monde et de son apparence, des leurres et de l'illusion et de la nudité des sentiments.

Si le Prince de bois ne témoigne guère de l'état de la scène hongroise au cour des années dix du siècle dernier, il porte néanmoins la trace des débats menés au sein de l'avant-garde hongroise. Il se fait écho, notamment, des discussions promues au sein des revues Nyugat et Ma, des peintres du Groupe des Huit ou des penseurs du Cercle du dimanche (fondé notamment par György Lukács et Béla Balázs). On y note une même prédisposition pour la recherche de la « nature essentielle des choses ", pour la rudesse des sentiments et la violence des affects, la prédominance de la nécessité intérieure sur une prétendue objectivité. En aval ou en amont d'auteurs tels Tamás Kóbor (Budapest), Karinthy (Solitude), Jenő Rejtö (nouvelles) ou, Kosztolányi (Esti Kornél), Bartók exprime une interrogation sur l'identité et la moralité au sein d'une société en désintégration ou jugée comme telle. Il révèle que la découverte de soi est intimement liée à celle de l'Autre, montrant l'une des plus belles qualités humaines : le souci de l'altérité. Toutes ses œuvres scéniques - Le Château de Barbe-Bleue, Le Prince de bois et Le Mandarin merveilleux - insistent sur l'un ou l'autre de ces aspects.

En tant que lecteur et admirateur de Nietzsche, Bartók entend, enfin, traiter les passions les plus brûlantes, et montrer dans le même temps une volonté de distanciation et de détachement liée à une conception vitaliste. Le format du conte lui offre cette possibilité de contempler les émotions et les analyser, pour mieux les vivre pleinement. À l'instar du philosophe allemand, il s'intéresse aux « émotions complexes » qui reflètent, seules, la réalité de la vie. Celles-ci diffèrent des sentiments entendus au sens commun et habituel du terme, car elles relèvent plutôt de la compréhension de l'intérieur des réalités de ce monde. La musique, à l'image des trois passages surlignés par le musicien dans le Prince de bois, doit ainsi avoir pour objectif d'explorer de l'intérieur la véracité des affects - des plus gais comme des plus tristes -, et de les révéler au spectateur. «L'art doit transmettre des chocs émotionnels et faire ainsi éprouver les grandes sensations de l'existence » écrit-il à Stefi Geyer, dans une lettre datée du 27 juillet 1907. Le Prince de bois est tributaire de cette pensée. Avec lui se referme une décennie décisive, où Bartók a pu s'émanciper d'un milieu jugé académique, en proposant un art hongrois nouveau et authentique, tout en prenant sa part au sein des débats questionnant l'avant-garde hongroise et ainsi s'inscrire dans une modernité sans compromis, bien que sans rupture avec le passé. Trouver, en d'autres termes, sa place d'artiste et de créateur - celui d'un art profond, vrai et sincère, et par là-même singulièrement émouvant. 


\section{Notes}

1 Lire notamment l'article de Péter Hanák «L'opérette viennoise et budapestoise dans l'histoire de la culture » et celui de Zsuzsa Radnóti « L'âge d'or de l'art dramatique hongrois », in Théâtre hongrois : d'une fin de siècle à l'autre, 1901-2001, sous la direction d'Anna Lakos, Montpellier, Maison Antoine Vitez, collection Les Cahiers, éd. Climats, 2001 - articles dont le présent paragraphe tire beaucoup de sa substance.

2 in Péter Hanák «L'opérette viennoise et budapestoise dans l'histoire de la culture », op. cit. page 41

3 Voir à cet égard l'article de Rósza Kocsis « Les tendances de l'avant-garde théâtrale dans la Hongrie de l'entre-deux guerres » in Cahiers du centre recherche sur le surréalisme, Mélusine 15 : le surréalisme en Hongrie, pages 215 sq.

4 Cf. L'excellent article de Edit Erdödy, « Sur la scène de l'âme. Les mystères de Béla Balázs » in Revue d'Etudes Françaises n'9 (2004), page 192

5 Cf. Edit Erdödy, op. cit. page 193

6 Idem. Page 196. Edit Erdödy mentionne également le fait que Balázs a assisté en 1908 à une représentation de Pelléas et Mélisande de Claude Debussy.

7 Voir la présentation passionnante de ces pièces par Pascale Alexandre-Bergues in Maurice Maeterlinck : L'intruse \& Intérieur, éd. Slatkine, Genève, 2005. Le théâtre de Maeterlinck y est présenté notamment comme une « dramaturgie de mort », un « théâtre de l'attente, du visible et de l'invisible » et un « tragique quotidien ». Je lui emprunte une partie de ses observations.

8 Cf. Pascale Alexandre-Bergues, op. cit., page 49

9 cf. Edit Erdödy, op. cit., page 192

10 Ibid.

11 Les appels sont mêmes numérotés dans la partition.

12 On rappellera que Le Mandarin merveilleux, autre conte, bénéficie aussi d'une structure par trois : les trois voyous, les trois hommes, les trois tentatives de séduction, les trois tentatives de meurtre, etc.

13 Béla Balázs, cité par György Kroó, « Ballet : The Wooden Prince », in The Bartók Companion, edited by Malcom Gillies, Faber and Faber, London, 1993, pages 362-63

14 cf. Sándor Ferenczi, «La psychologie du conte », in Sándor Ferenczi, Psychanalyse, t. II, CEuvres complètes 1913-1919, éd. Payot, Paris, 1970

15 Sándor Ferenczi, «Le Développement du sens de réalité et ses stades » In Euvres Complètes, op. cit., Payot, 1970, pages 51-65

16 Ibid., p. 65

17 Béla Bartók, «Le Prince de bois, opus 13 », 1917, in Béla Bartók : Ecrits, édités par Philippe Albèra et Peter Szendy, éd. Contrechamps, Genève, 2006, pages 77-78

18 Lire Zoltán Kodály : «Un opéra de Béla Bartók » in Bartók, sa vie et son æuvre, publié sous la direction de Bence Szabolcsi, éd. Boosey\&Hawkes, Paris, 1968, page 72 notamment

19 Poco lento, piu lento, Lento ; la noire passe graduellement de 100 à 92, 80, puis 66-60 